\title{
THE SUB-PARSEC SCALE JETS OF AGN
}

\author{
T.P. KRICHBAUM, W. ALEF, A. WITZEL \\ Max-Planck-Institut für Radioastronomie, Bonn, Germany
}

With an angular resolutions of $0.05-0.2$ mas, millimeter-VLBI ${ }^{1}$ observations (at 22,43 , and $86 \mathrm{GHz}$ ) allow to investigate the very central sub-parsec scale - regions of active galactic nuclei (AGN), which are selfabsorbed at lower frequencies. Here we briefly present preliminary results from recent observations of Cygnus $\mathrm{A}$ at $22 \& 43 \mathrm{GHz}$, which reveal evidence for subluminal motion in jet and counter-jet, and $86 \mathrm{GHz}$ VLBI observations of two extreme $\gamma$-blazars, suggesting a tight correlation between their $\gamma$-ray activity and the generation of jets.

Cygnus A: Its proximity ( $z=0.057)$ and large radio luminosity makes the archetypical FR II radio galaxy Cygnus A particularly interesting for high spatial resolution imaging. The detection of motion in jet and counterjet will allow - at least in principle - to determine the orientation of the jet, its intrinsic velocity and the cosmological distance of Cygnus A. After initial detection with VLBI at $43 \mathrm{GHz}$ (Krichbaum et al., 1993, AA 275, 375), we obtained an improved second epoch $43 \mathrm{GHz}$ VLBI image. From this and $22 \mathrm{GHz}$ observations performed in parallel, a two-sided core-jet structure is revealed (Fig.1). We tentatively determined the position of the VLBIcore using its compactness and inverted spectrum. With this identification, we find subluminal motion with velocities in the range of $v / c \simeq 0.1-0.3$ $\left(H_{0}=100 \mathrm{~km} \mathrm{~s}^{-1} \mathrm{Mpc}^{-1}, q_{0}=0.5\right)$ for at least 4 distinct jet components located at $r \leq 3-4$ mas core separation (Krichbaum et al., 1996, Greenbank workshop, eds. Carilli \& Harris, p.92). At $5 \mathrm{GHz}$ slightly higher velocities in the range $0.2 \leq v / c \leq 0.5$ for components located at larger core separations $(r>5$ mas, Carilli et al., 1994, AJ 108, 64) are found and indicate either apparent acceleration along the jet or the presence of pattern speeds. From the $22 \mathrm{GHz}$ maps (Fig.1) and from an unpublished map at $18 \mathrm{~cm}$ (Fig.2) we obtained a jet to counter-jet ratio in the range $R \simeq 2-8$.

0528+134: Two $3 \mathrm{~mm}$ VLBI-maps (1993.26 \& 1994.0) reveal strong evidence for the emergence of a new feature east of the main component (Fig.2). Since at larger core separations $(r>1$ mas) the jet is oriented to the north (eg. Zhang et al., 1994, ApJ 432, 91), motion along a strongly bent path $\left(\Delta\right.$ p.a. $\simeq 90^{\circ}$ for $r \leq 1$ mas) is suggested. Back-extrapolation of the motion $\left(\beta_{a p p} \simeq 5\right)$ of the new component yields strong evidence that it was 

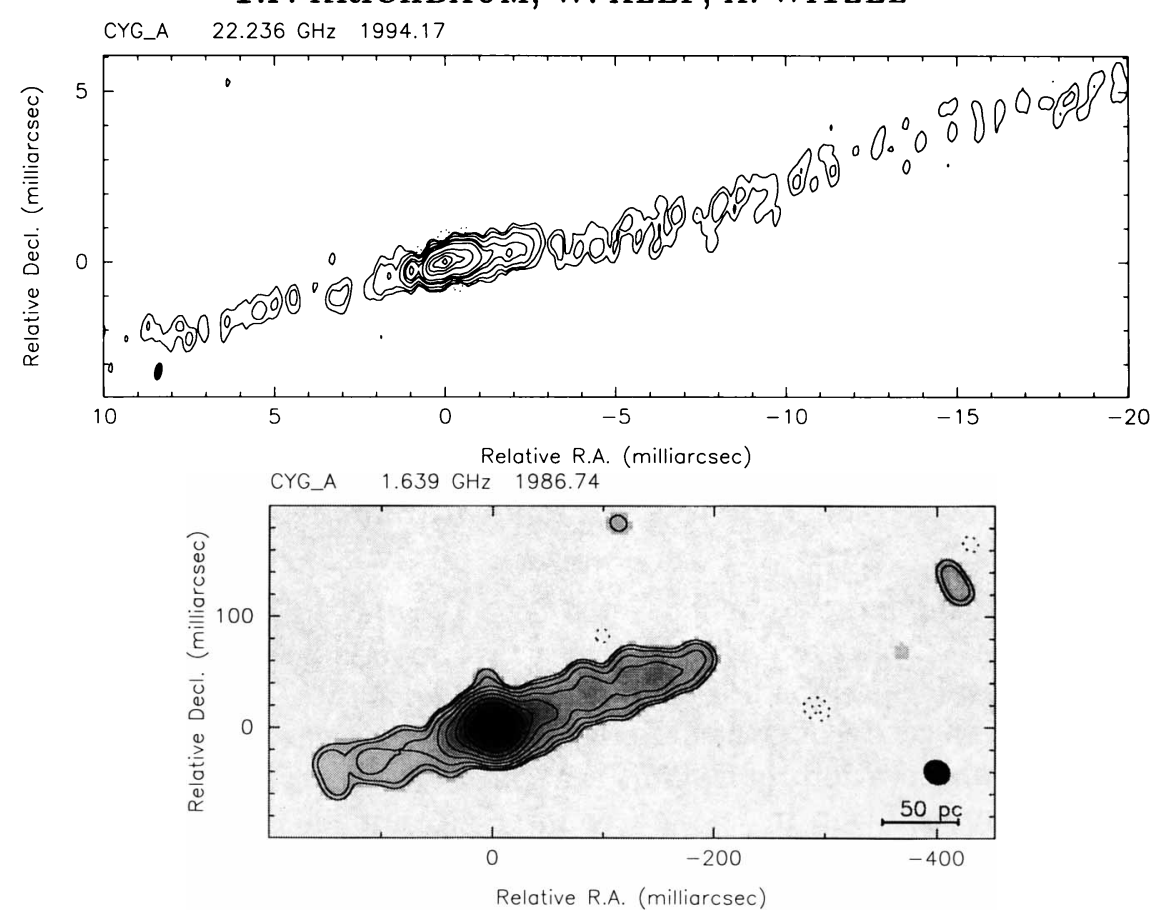

Figure 1. Top: Jet- and counter-jet of Cygnus A at $22 \mathrm{GHz}$ (1994.17). Only the central part of a larger map is shown. Contour levels are $-0.1,0.1,0.3,1,3,5,10,30,50,70,90 \%$ of the peak. The restoring beam size is $0.5 \times 0.2 \mathrm{mas}$, p.a. $=-11^{\circ}, 0.2$ mas correspond to 0.15 pc. Bottom: EVN-VLBI map of Cygnus $A$ at $1.6 \mathrm{GHz}$. Contour levels are $-0.5,-0.3$, $0.3,0.5,1,2,4,8,16,32,64 \%$ of the peak, the beam size is $23.6 \times 20.7$ mas, p.a. $=54^{\circ}$. The jet is oriented along P.A. $\simeq 287^{\circ}$, well aligned with the kpc-jet. From this map a jet-to-counterjet ratio of $R \simeq 2$ is obtained. The bar indicates a linear scale of $50 \mathrm{pc}$.

ejected during the correlated $\mathrm{mm}-/ \gamma$-ray outburst of 1993.5 (Krichbaum, et al., 1996, Proc. Nat. Acad. Sci. USA, in press). This supports the idea that the $\gamma$-radiation originates from highly relativistic jets (eg. Sikora et al. , 1994, ApJ 421, 153).

3C 454.3: From two $3 \mathrm{~mm}$ VLBI-maps (1993.26 \& 1994.0) an evolving complex core-jet structure is revealed (Fig.3), showing superluminal motion with $\beta_{a p p} \simeq 6-7$ at $r \leq 1$ mas. This is considerably slower than the motion reported by Pauliny-Toth et al. (in preparation) at larger core separations, who find component acceleration from $\beta_{a p p} \simeq 8$ at $r=2$ mas to $\beta_{a p p} \simeq 21$ at $r=5$ mas. If interpreted geometrically, an ultra-relativistic flow $(\gamma \simeq 20)$ along a spatially bent path and differential Doppler-boosting (eg. like in $3 \mathrm{C} 345$, Zensus, this conference) must be considered.

VLBI imaging at $86 \mathrm{GHz}$ therefore can provide important tests of such geometrical interpretation schemes and will reveal - owing to its high angular and spatial resolution - early detections of new jet components, allowing to study the relation between jet production and broad-band flux density activity (eg. Aller et al.\& Romanova et al., this conference). 

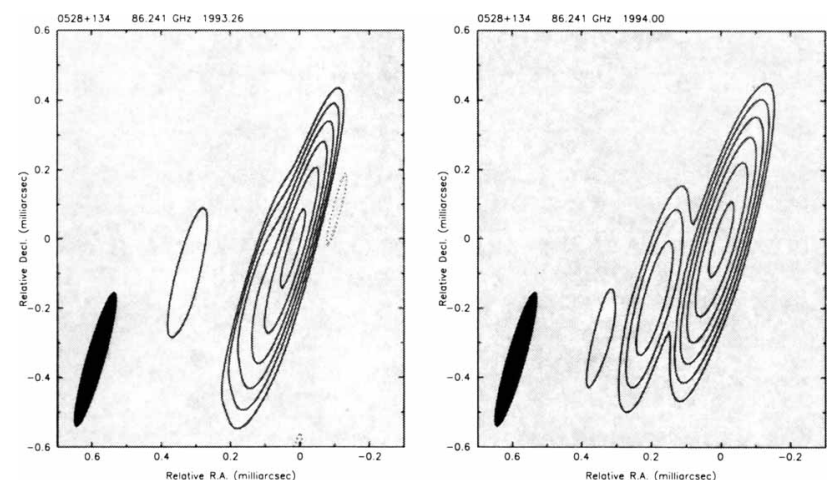

Figure 2. The evolution of the sub-mas structure of $0528+134$ at $86 \mathrm{GHz}$ between 1993.26 (left) and 1994.00 (right). The restoring beam size is $50 \times 400 \mu \mathrm{as}$, p.a. $=-16^{\circ}$. Contour levels in both maps are $-2.5,2.5,5,10,20,40,80 \%$ of the peak flux density of 0.68 (left) respectively 0.65 (right) $\mathrm{Jy} /$ beam. Note that for unknown reasons the flux densities seen in both maps are by a factor $\sim 3$ lower than the total flux density. The maps and Gaussian modelfits to the data reveal the ejection of a new component from the compact selfabsorbed core.
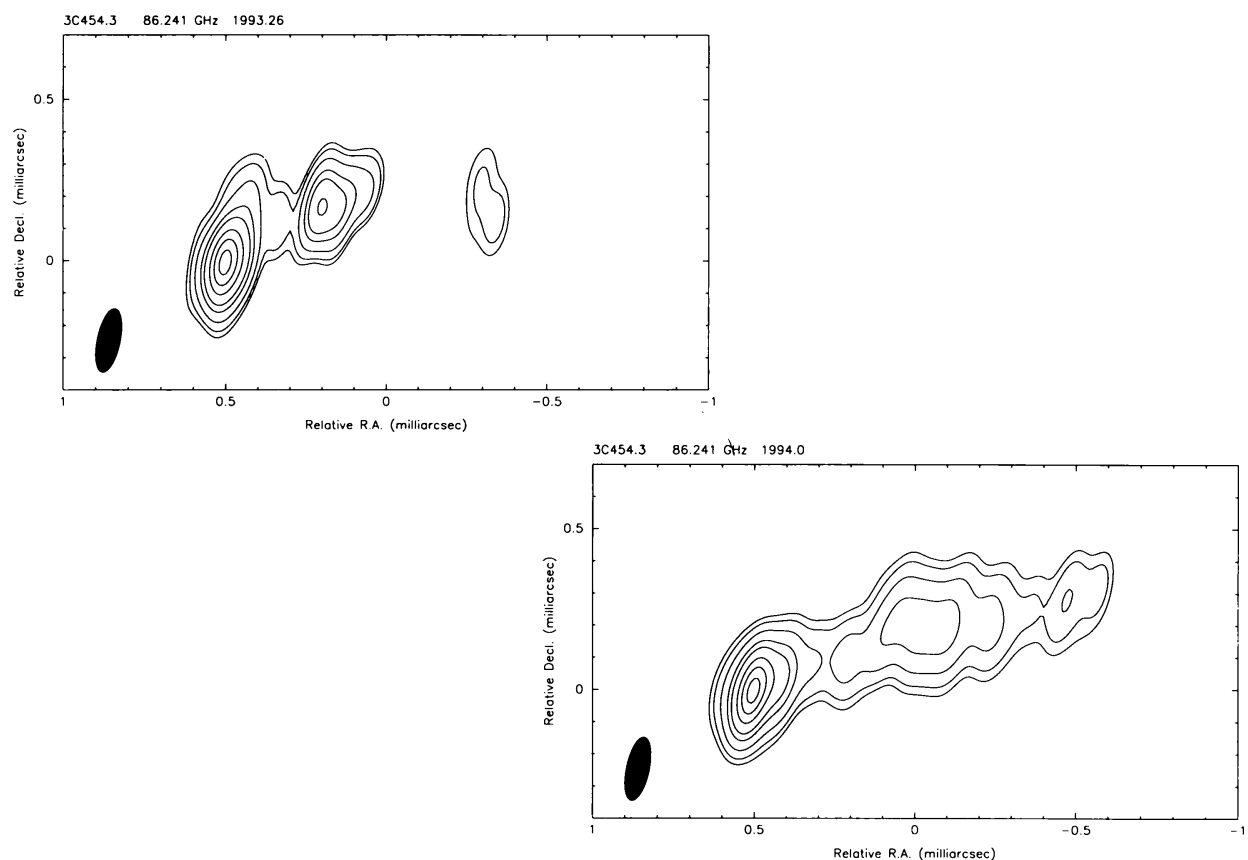

Figure 3. Clean-maps of $3 \mathrm{C} 454.3$ at $86 \mathrm{GHz}$ obtained in 1993.26 (top) and 1994.0 (bottom). Both maps are convolved with a beam of size $70 \times 200 \mu$ as, p.a. $=-12^{\circ}$. Contour levels are $-3,3,5,10,20,30,50,70,90 \%$ of the peak flux density of $0.94 \mathrm{Jy} /$ beam (top) and $1.35 \mathrm{Jy} /$ beam (bottom). Next to the stationary assumed bright and compact core component located at the map center, two features located at $r \simeq 0.3$ mas and $r \simeq 0.8 \mathrm{mas}$ (in 1993.26) seem to separate from the core with $\beta_{a p p} \simeq 6-7$.

${ }^{1} \mathrm{~mm}-\mathrm{VLBI}$ is a joint effort of the observatories at Effelsberg, Onsala \& SEST, Pico Veleta, Haystack, Quabbin, Kitt Peak, Owens Valley, Hat Creek and the VLBA. T.P.K. appreciates support of the BMFT-Verbundfoschung. 